\title{
BMR
}

\section{Correlations between the COMT gene rs4680 polymorphism and susceptibility to ovarian cancer}

\author{
W. Pan ${ }^{1}$ and H. Liao ${ }^{2}$ \\ 1Department of Obstetrics and Gynecology, Huangshi Central Hospital of Hubei \\ Province (Affiliated Hospital of Hubei Polytechnic University), Hubei Key laboratory \\ of Kidney Disease Pathogenesis and Intervention, Huangshi, China \\ ${ }^{2}$ Department of Pharmacy, Huangshi Central Hospital of Hubei Province (Affiliated \\ Hospital of Hubei Polytechnic University), Hubei Key Laboratory of Kidney Disease \\ Pathogenesis and Intervention, Huangshi, China \\ Corresponding author: H. Liao \\ E-mail: Liao71Hua@163.Com \\ Genet. Mol. Res. 14 (4): 16813-16818 (2015) \\ Received July 4, 2015 \\ Accepted September 20, 2015 \\ Published December 14, 2015 \\ DOI http://dx.doi.org/10.4238/2015.December.14.8
}

ABSTRACT. The objective of this study was to perform a systematic review of the correlations between the single nucleotide polymorphism rs 4680 in the catechol-O-methyltransferase (COMT) gene and susceptibility to ovarian cancer. A computer search was carried out for relevant case-control studies published between January 2000 to January 2014 in databases such as Ovid, EBSCO, PubMed, CNKI, CBMDISC, VIP, and WanFang Data. The literature was screened based on inclusion and exclusion criteria. A meta-analysis was performed by calculating the combined odds ratios (OR) and $95 \%$ confidence intervals $(\mathrm{Cl})$ using the RevMan 5.0. A total of 7 case-control studies were selected, which included 1439 cases and 2927 control subjects. Meta-analysis showed that the rs4680 polymorphism was not associated with ovarian cancer [GG vs $(\mathrm{GA}+\mathrm{AA})$ : $\mathrm{OR}=1.02$, $95 \% \mathrm{Cl}=0.88-1.19 ; \mathrm{G}$ vs A allele: $\mathrm{OR}=1.0,95 \% \mathrm{Cl}=0.90-1.11]$. We, 
therefore, conclude that the COMT rs4680 polymorphism is not associated with susceptibility to ovarian cancer.

Key words: COMT; Ovarian cancer; Single nucleotide polymorphism; Systematic review; Meta-analysis; Case-control study

\section{INTRODUCTION}

Ovarian cancer is one of the most common female malignancies, and is the leading cause of death among gynecological cancers (Hennessy et al., 2009). Over 205,000 new cases and 125,000 deaths are due to ovarian cancer (Jemal et al., 2011). In terms of etiology, polymorphisms within genes responsible for estrogen catabolism are known to alter cellular levels of genotoxic 4-hydroxylated catechol estrogens and antiangiogenic 2-methoxyestradiol, thus influencing risk of developing ovarian cancers (Holt SK et al., 2007).

The catechol-O-methyltransferase (COMT) gene is located on chromosome 22q11.2 and consists of six exons, with exons 1 and 2 being noncoding (Park et al., 2009). The rs4680 polymorphism is a G-to-A transition at codon 158 in exon 4 that leads to a substitution of methionine for valine; this results in a thermolabile enzyme with reduced activity (Lachman et al., 1996). At the time of writing, several case-control studies on the relationship between rs4680 and susceptibility to ovarian cancers have been published (Goodman et al., 2000; Goodman et al., 2001; Garner et al., 2002; Delort et al., 2008). However, these studies have certain limitations, including variations in research quality, small sample size, or different regional and ethnic backgrounds of the subjects. Thus, the conclusions drawn from these studies are not very firm and have limited credibility. Our study aimed to perform a meta-analysis of the relationship between rs 4680 polymorphism and susceptibility to ovarian cancers by using data from case-control studies in China and other countries. The results from this study will provide more reliable evidence for basic research and clinical treatment into this devastating disease.

\section{MATERIAL AND METHODS}

We included projects that were designed as case-control studies in order to assess the association between rs4680 polymorphism and susceptibility to ovarian cancers. Among studies published by the same authors, those with the highest quality and largest sample size were selected. Only those that reported proper statistical methods and yielded highly reliable data, definitive results, genotype data, $\mathrm{OR}$ and $95 \% \mathrm{Cl}$ (or where such values could be calculated from the original data) were included. Studies that were not on human subjects were excluded.

\section{Search strategy}

A Medical Subject Headings (MeSH) method was used to retrieve studies from databases. "COMT" or "catechol-O-methyltransferase" and "1222 G > A" or "Val158Met" or "rs4680" and "polymorphism" and "ovarian cancer" were used as keywords. Studies published between January 2000 and January 2014 were searched. 


\section{Data retrieval}

Two investigators assessed the quality of the studies and retrieved data from the literature based on the same quality standards, and then performed a cross-check. Any disagreement was resolved by discussion or by a third investigator.

\section{Statistical analysis}

Meta-analysis was performed using the RevMan 5.0 software. Cochran's $Q$ test was used for the analysis of heterogeneity between the results of each study. When there was no heterogeneity between studies, a fixed-effect model was used for the meta-analysis. When there was heterogeneity, a random-effect model was used for the meta-analysis. The OR and $95 \% \mathrm{Cl}$ of each allele and genotype frequency were calculated for each study. The Hardy-Weinberg equilibrium of the control group was calculated. $\mathrm{P}<0.05$ was considered to be statistically significant. Sensitivity analysis was conducted using the individual exclusion method. The overall effects were re-assessed and compared with the overall effects prior to exclusion. Begg and Egger tests were applied to determine whether there was publication bias in the studies.

\section{RESULTS}

\section{Literature search and quality evaluation}

Fifty-four papers were initially retrieved. After screening based on the criteria outlined in the Methods, seven case-control studies were examined. This included 1439 cases and 2927 control subjects. The basic characteristics of the cases included are shown in Table I. All the 7 studies (Goodman et al., 2000; Goodman et al., 2001; Garner et al., 2002; Sellers et al., 2005; Holt et al., 2007; Delort et al., 2008; Jakubowska et al., 2010) contained allelic data. All studies were consistent with the Hardy-Weinberg equilibrium.

\section{Table 1. Characteristics of the studies included.}

\begin{tabular}{|c|c|c|c|c|c|c|c|c|c|c|}
\hline \multirow[t]{2}{*}{ Authors } & \multirow[t]{2}{*}{ Publication year } & \multirow[t]{2}{*}{ Ethnicity } & \multicolumn{3}{|c|}{ Case group } & \multicolumn{3}{|c|}{ Control group } & \multirow[t]{2}{*}{ Genotyping methods } & \multirow[t]{2}{*}{ HWE } \\
\hline & & & GG & GA & AA & GG & GA & AA & & \\
\hline Jakubowska et al. & 2010 & Caucasian & 27 & 82 & 37 & 67 & 164 & 49 & PCR-RFLP & Yes \\
\hline Delort et al. & 2008 & Caucasian & 11 & 22 & 18 & 237 & 480 & 283 & TaqMan & Yes \\
\hline Holt et al. & 2007 & African and Caucasian & 91 & 139 & 80 & 156 & 267 & 153 & TaqMan & Yes \\
\hline Garner et al. & 2002 & Caucasian & 59 & 103 & 48 & 52 & 119 & 54 & PCR-RFLP & Yes \\
\hline Goodman et al. & 2001 & Caucasian & 52 & 57 & 16 & 68 & 57 & 19 & PCR-RFLP & Yes \\
\hline Goodman et al. & 2000 & Caucasian & 27 & 54 & 27 & 25 & 52 & 29 & PCR-RFLP & Yes \\
\hline Sellers et al. & 2005 & African and Caucasian & 129 & 241 & 119 & 150 & 299 & 147 & PCR-RFLP & Yes \\
\hline
\end{tabular}

\section{Meta-analysis}

Our initial analysis revealed no heterogeneity among the studies $\left(I^{2}=0 \%, P=0.58\right)$. Therefore, a fixed effects model for pooled analysis was applied. The GG genotype was defined as the exposure factor, and the AA genotype was defined as the non-exposure factor. The metaanalysis showed no significant difference between the GG genotype and the GA + AA genotype in terms of ovarian risk $[\mathrm{OR}=1.02,95 \% \mathrm{Cl}(0.88,1.19), \mathrm{P}=0.76]$ (Figure 1). The meta-analysis 
showed that people with the $G$ allele did not have increased susceptibility to ovarian cancer compared to people with the A allele $[\mathrm{OR}=1.0,95 \% \mathrm{Cl}(0.9,1.10), \mathrm{P}=0.93]$ (Figure 2).

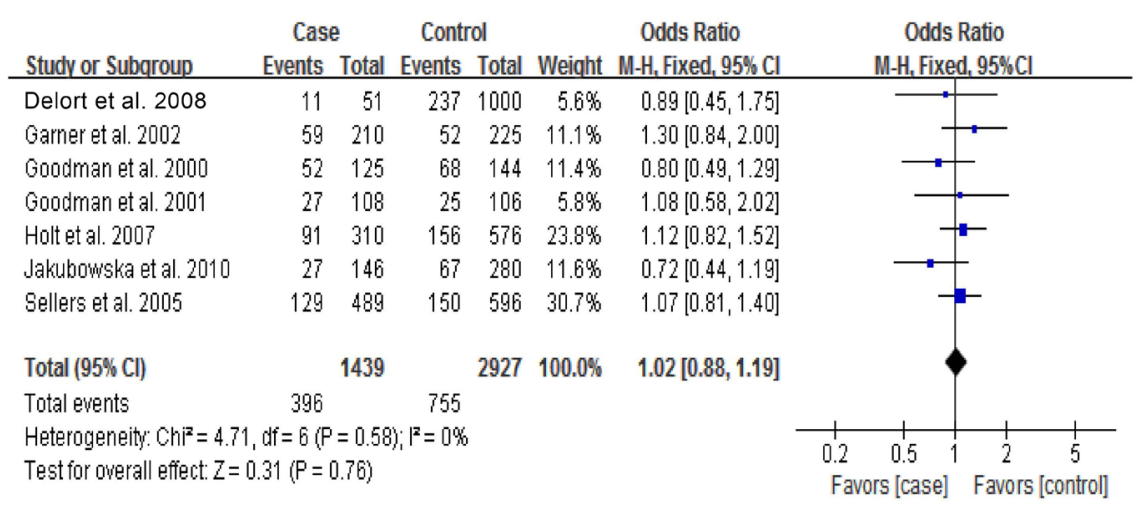

Figure 1. Forest plot of ovarian cancer susceptibility and the rs 4680 polymorphism of COMT (GG vs GA+AA). The horizontal lines correspond to the study-specific OR and $95 \% \mathrm{Cl}$, respectively. The area of the squares reflects the study-specific weight. The diamond represents the pooled results of OR and $95 \% \mathrm{Cl}$.

\begin{tabular}{|c|c|c|c|c|c|c|c|}
\hline Study or Subqroup & $\begin{array}{r}\text { Case } \\
\text { Events }\end{array}$ & Total & $\begin{array}{l}\text { Contr } \\
\text { Events }\end{array}$ & $\begin{array}{l}\text { rol } \\
\text { Total }\end{array}$ & Weight & $\begin{array}{c}\text { Odds Ratio } \\
\text { M-H, Fixed, 95\% Cl }\end{array}$ & $\begin{array}{c}\text { Odds Ratio } \\
\text { M-H, Fixed, 95\%C }\end{array}$ \\
\hline Delort et al. 2008 & 44 & 102 & 954 & 2000 & $6.3 \%$ & $0.83[0.56,1.24]$ & \\
\hline Garner et al. 2002 & 221 & 420 & 223 & 450 & $12.2 \%$ & $1.13[0.87,1.48]$ & \\
\hline Goodman et al. 2000 & 161 & 250 & 193 & 288 & $7.6 \%$ & $0.89[0.62,1.27]$ & \\
\hline Goodman et al. 2001 & 108 & 216 & 102 & 212 & $6.1 \%$ & $1.08[0.74,1.58]$ & \\
\hline Holt et al. 2007 & 321 & 620 & 579 & 1152 & $23.3 \%$ & $1.06[0.87,1.29]$ & \\
\hline Jakubowska et al. 2010 & 136 & 292 & 298 & 560 & $13.0 \%$ & $0.77[0.58,1.02]$ & \\
\hline Sellers et al. 2005 & 499 & 978 & 599 & 1192 & $31.5 \%$ & $1.03[0.87,1.22]$ & \\
\hline Total (95\% Cl) & & 2878 & & 5854 & $100.0 \%$ & $1.00[0.90,1.10]$ & \\
\hline Total events & 1490 & & 2948 & & & & \\
\hline \multicolumn{7}{|c|}{$\begin{array}{l}\text { Heterogeneity. } C h i^{2}=6.05, d f=6(P=0.42) ; I^{2}=1 \% \\
\text { Test for overall effect: } Z=0.09(P=0.93)\end{array}$} & 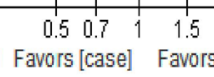 \\
\hline
\end{tabular}

Figure 2. Forest plot of ovarian cancer susceptibility and the rs4680 polymorphism of COMT (allele model: $\mathrm{G}$ vs A). The horizontal lines correspond to the study-specific OR and $95 \% \mathrm{Cl}$, respectively. The area of the squares reflects the study-specific weight. The diamond represents the pooled results of OR and $95 \% \mathrm{Cl}$.

\section{Sensitivity analysis}

The individual exclusion method was used for the sensitivity analysis in both the GG and AA genotypes. The smallest combined OR after exclusion was $0.77[95 \% \mathrm{Cl}(0.58,1.02)]$, and the largest combined OR after exclusion was $1.13[95 \% \mathrm{Cl}(0.87,1.48)]$. Comparisons of the meta-analysis results before and after this exclusion revealed no significant differences, indicating that the analysis exhibited relatively low sensitivity and that therefore the analysis results were relatively robust and credible.

\section{Publication bias}

Funnel plot and the Egger test were performed to assess the publication bias of the literature. The Egger test further confirmed the absence of publication bias in this meta-analysis $(P>0.05$; Figure 3$)$. 


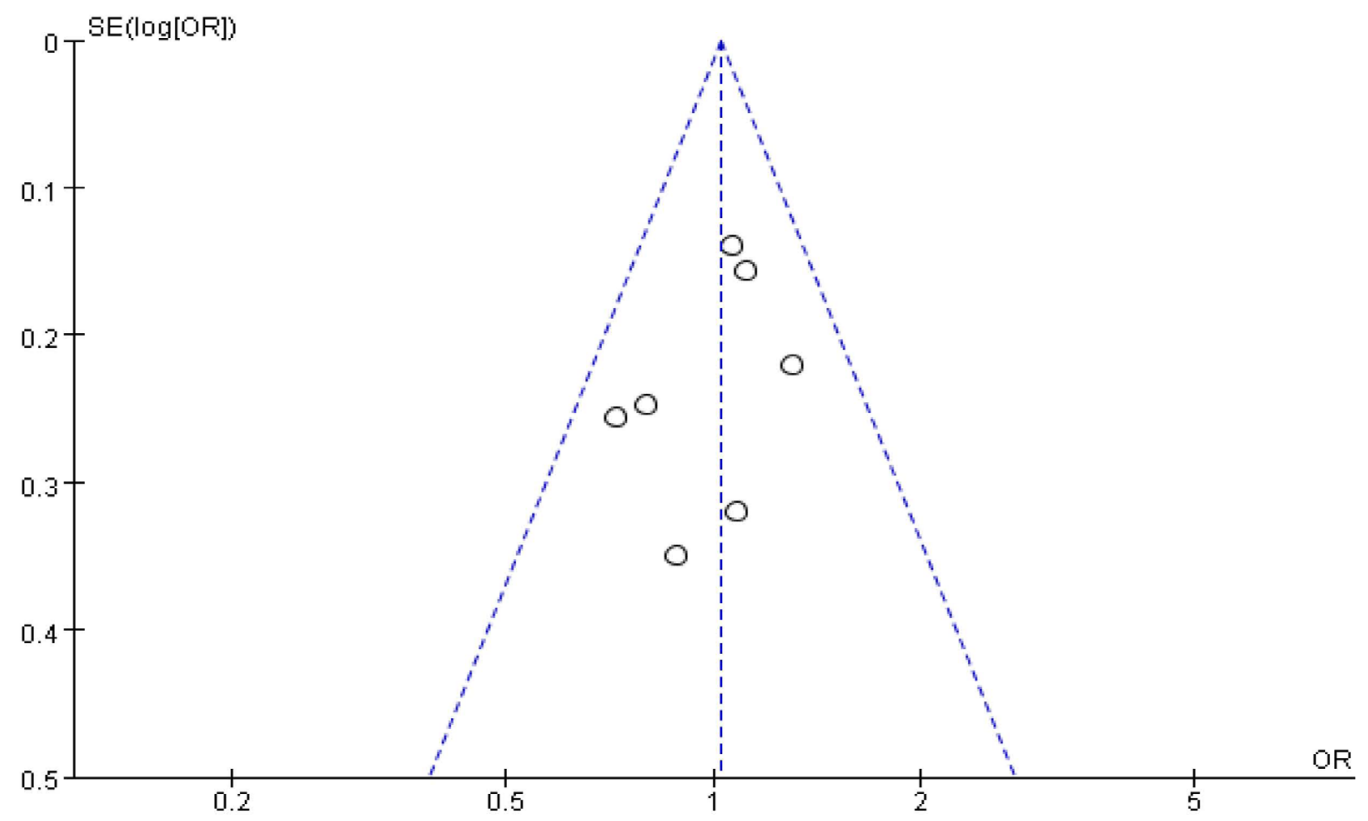

Figure 3. Begg's funnel plot for publication bias tests. Each point represents a separate study for the indicated association. Log OR represents natural logarithm of OR. Vertical line represents the mean effect size.

\section{DISCUSSION}

In this study, we performed a systematic review of the associations between COMT polymorphisms and susceptibility to ovarian cancer. Our results indicated that at the rs4680 locus, the OR of the relative susceptibility to ovarian cancer of the $\mathrm{G}$ allele compared to the A allele was $1.0[95 \% \mathrm{Cl}(0.90,1.10), \mathrm{P}=0.93]$ and therefore was not statistically significant. These results indicate that the $\mathrm{G}$ allele does not increase susceptibility to ovarian cancer.

Catechol-O-methyltransferase is a phase II enzyme that is involved in the conjugation and inactivation of catechol estrogens (Axelrod et al., 1958). COMT catalyzes the methylation of catechol estrogens to less polar monomethyl ethers. O-Methylation increases the concentrations of 4-methoxyestradiol (4-MeOE2) and 2-methoxyestradiol (2-MeO-E2): 2-MeO-E2 possesses antiproliferative, cytotoxic, and apoptotic activity. By inducing cell death, this metabolite eliminates cells with otherwise potentially harmful DNA damage (Dawling et al., 2001). Allelic variation in COMT is likely related to enzymatic activity. However, we observed no evidence for an increased risk of ovarian cancer associated with the COMT Val158Met polymorphism.

We found no heterogeneity among the studies we examined. Furthermore, funnel plot of the Begg test revealed no publication bias. These observations strengthen the results of the current analysis. However, our study does have limitations, since it is restricted to the analysis of studies in only the Chinese and English languages. Therefore, potential publication and language biases could interfere with the meta-analysis. 


\section{REFERENCES}

Axelrod J and Tomchick R (1958). Enzymatic O-methylation of epinephrine and other catechols. J. Biol. Chem. 233: 702-5.

Dawling S, Roodi N, Mernaugh RL, Wang X, et al. (2001). Catechol-O-methyltransferase (COMT)-mediated metabolism of catechol estrogens, comparison of wild-type and variant COMT isoforms. Cancer Res. 61: 6716-22.

Delort L, Chalabi N, Satih S, Rablau N, et al. (2008). Association between genetic polymorphisms and ovarian cancer risk. Anticancer Res. 28: 3079-81.

Garner El, Stokes EE, Berkowitz RS, Mok SC, et al. (2002). Polymorphisms of the estrogen-metabolizing genes CYP17 and catechol-Omethyltransferase and risk of epithelial ovarian cancer. Cancer Res. 62: 3058-62.

Goodman JE, Lavigne JA, Hengstler JG, Tanner B, et al. (2000).Catechol-O-methyltransferase polymorphism is not associated with ovarian cancer risk. Cancer Epidemiol Biomarkers Prev. 9: 1373-6.

Goodman MT, McDuffie K, Kolonel LN, Terada K, et al. (2001). Casecontrol study of ovarian cancer and polymorphisms in genes involved in catecholestrogen formation and metabolism. Cancer Epidemiol Biomarkers Prev. 10: 209-16.

Hennessy BT, Coleman RL and Markman M (2009). Ovarian cancer. Lancet 374: 1371-82.

Holt SK, Rossing MA, Malone KE, Schwartz SM, et al. (2007). Ovarian cancer risk and polymorphisms involved in estrogen catabolism. Cancer Epidemiol Biomarkers Prev. 16: 481-9.

Jakubowska A, Gronwald J, Menkiszak J, Gorski B, et al. (2010). BRCA1-associated breast and ovarian cancer risks in Poland, no association with commonly studied polymorphisms. Breast. Cancer Res. Treat. 119: 201-11.

Jemal A, Bray F, Center MM, Ferlay J, et al. (2011). Global cancer statistics. CA Cancer J. Clin. 61: 69-90.

Lachman HM, Papolos DF, Saito T, Yu YM, et al. (1996). Human catechol-O-methyltransferase pharmacogenetics, description of a functional polymorphism and its potential application to neuropsychiatric disorders. Pharmacogenetics 6: 243-50.

Park BL, Shin HD, Cheong HS, Park CS, et al. (2009). Association analysis of COMT polymorphisms with schizophrenia and smooth pursuit eye movement abnormality. J. Hum. Genet. 54: 709-12.

Sellers TA, Schildkraut JM, Pankratz VS, Vierkant RA, et al. (2005). Estrogen bioactivation, genetic polymorphisms, and ovarian cancer. Cancer Epidemiol Biomarkers Prev. 14: 2536-43. 